\title{
PD-1 blockade during chronic SIV infection reduces hyperimmune activation and microbial translocation in rhesus macaques
}

\author{
Ravi Dyavar Shetty, ${ }^{1}$ Vijayakumar Velu, ${ }^{1}$ Kehmia Titanji, ${ }^{1}$ Steven E. Bosinger, ${ }^{1}$ \\ Gordon J. Freeman,2 Guido Silvestri,1,3 and Rama Rao Amara'1,4 \\ ${ }^{1}$ Yerkes National Primate Research Center, Emory Vaccine Center, Emory University, Atlanta, Georgia, USA \\ 2Department of Medical Oncology, Dana-Farber Cancer Institute, Harvard Medical School, Boston, Massachusetts, USA \\ ${ }^{3}$ Department of Pathology and ${ }^{4}$ Department of Microbiology and Immunology, Emory University, Atlanta, Georgia, USA
}

\begin{abstract}
Hyperimmune activation is a strong predictor of disease progression during pathogenic immunodeficiency virus infections and is mediated in part by sustained type I IFN signaling in response to adventitious microbial infection. The immune inhibitory receptor programmed death-1 (PD-1) regulates functional exhaustion of virus-specific $\mathrm{CD8}^{+} \mathrm{T}$ cells during chronic infections, and in vivo $\mathrm{PD}-1$ blockade has been shown to improve viral control of SIV. Here, we show that PD-1 blockade during chronic SIV infection markedly reduced the expression of transcripts associated with type I IFN signaling in the blood and colorectal tissue of rhesus macaques (RMs). The effect of PD-1 blockade on type I IFN signaling was durable and persisted even under conditions of high viremia. Reduced type I IFN signaling was associated with enhanced expression of some of the junction-associated genes in colorectal tissue and with a profound decrease in plasma LPS levels, suggesting a possible repair of gut-associated junctions and decreased microbial translocation into the blood. PD-1 blockade enhanced immunity to gut-resident pathogenic bacteria, control of gut-associated opportunistic infections, and survival of SIV-infected RMs. Our results suggest PD-1 blockade as a potential novel therapeutic approach to enhance combination antiretroviral therapy by suppressing hyperimmune activation in HIV-infected individuals.
\end{abstract}

\section{Introduction}

The immune inhibitory receptor programmed death-1 (PD-1) plays an important role in regulating functional exhaustion of virus-specific $\mathrm{CD}^{+} \mathrm{T}$ cells during chronic infections $(1)$. Studies in mice demonstrated that PD-1 plays a critical role in determining the functionality of virus-specific $\mathrm{CD}^{+} \mathrm{T}$ cells in the control of chronic lymphocytic choriomeningitis virus (LCMV) infection (2). Later studies extended these observations to HIV/SIV-specific T cells in vitro (3-7). More recently, we $(8,9)$ and others $(10)$ have demonstrated that in vivo PD-1 blockade during chronic SIV infection restores the function of SIV-specific cellular and humoral immune responses and improves viral control. PD-1 and its ligands are expressed on many different immune cells, and thus in vivo blockade of PD-1 signaling could influence many immunoregulatory pathways. To gain insight into the influence of in vivo PD-1 blockade on these pathways during chronic SIV infection, we performed transcriptional profiling of more than 23,000 genes $(52,865$ probesets) expressed in colorectal mucosa (referred to hereafter as gut, a preferential site of virus replication) and blood of SIV-infected rhesus macaques (RMs) using RNA obtained at 14 days following in vivo blockade. For these analyses, we used samples obtained from a previously reported study (8) in which we treated 9 SIV-infected macaques with an antibody to human PD-1 and 5 SIV-infected macaques with a control antibody on days $0,3,7$, and 10 . Of the 9 PD- 1 antibody-treated animals, 5 received the antibody at 10 weeks (early chronic phase) and 4 received it at 90 weeks (late chronic phase) after infection.

Conflict of interest: Gordon J. Freeman has patents and receives patent royalties on therapies involving PD-1. Rama Rao Amara, Vijayakumar Velu, and Kehmia Titanji are co-inventors of PD-1 technology that has been licensed to Genetech Inc. by Emory University.

Citation for this article: J Clin Invest. 2012;122(5):1712-1716. doi:10.1172/JCI60612

\section{Results and Discussion}

Comparison of transcriptional profiles between PD-1 antibody$(n=5)$ and control antibody-treated $(n=3)$ SIV-infected RMs at 14 days following PD-1 blockade revealed significant changes (>1.5-fold change and $P<0.05)$ in 236 genes in the gut and 312 genes in blood. We then categorized these significantly altered genes in terms of their role in biological pathways using ingenuity pathway analysis (IPA). Consistent with our prior findings (8), IPA also revealed a significant upregulation of genes associated with TCR signaling (Figure 1A). However, we observed a significant downregulation of genes involved in type I IFN and IFN regulatory factor (IRF) activation signaling, which suggested a negative influence on these pathways and possible reduction of immune activation in PD-1 antibody-treated SIV-infected RMs.

Sustained high levels of type I IFN responses in blood, lymphoid, and gut tissue of pathogenic SIV-infected RMs have been implicated in immune activation and faster disease progression (11-13). Also, HIV-specific T cells from progressors compared with controllers express higher levels of type I IFN-responsive genes (14). We further looked into the individual genes involved in IFN signaling that were significantly modulated at 14 days following PD-1 blockade in our microarray analyses and found a number of type I IFN stimulatory genes (ISGs) to be downmodulated by about 2- to 4-fold following PD-1 blockade, in both blood and gut (Figure 1B and Supplemental Figure 1; supplemental material available online with this article; doi:10.1172/JCI60612DS1). Temporal microarray analysis of ISG expression before and after PD-1 blockade in two SIV-infected late chronic RMs also revealed a similar downregulation of many ISGs (Supplemental Figure 2). It is important to note that consistent with previous reports $(12,13)$, expression of all of these ISGs was greatly increased following SIV infection (Figure 1B). 
A

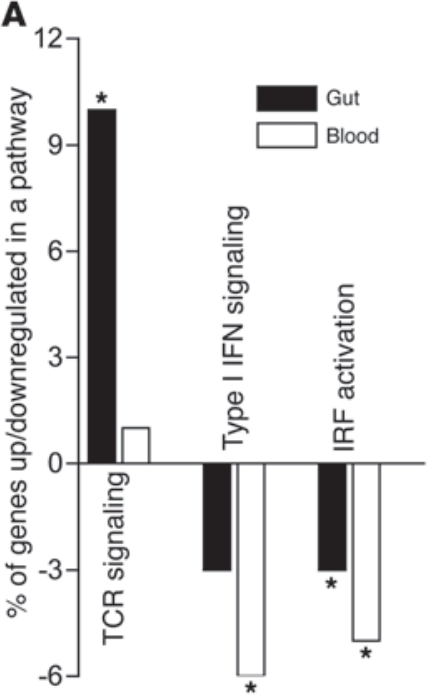

c

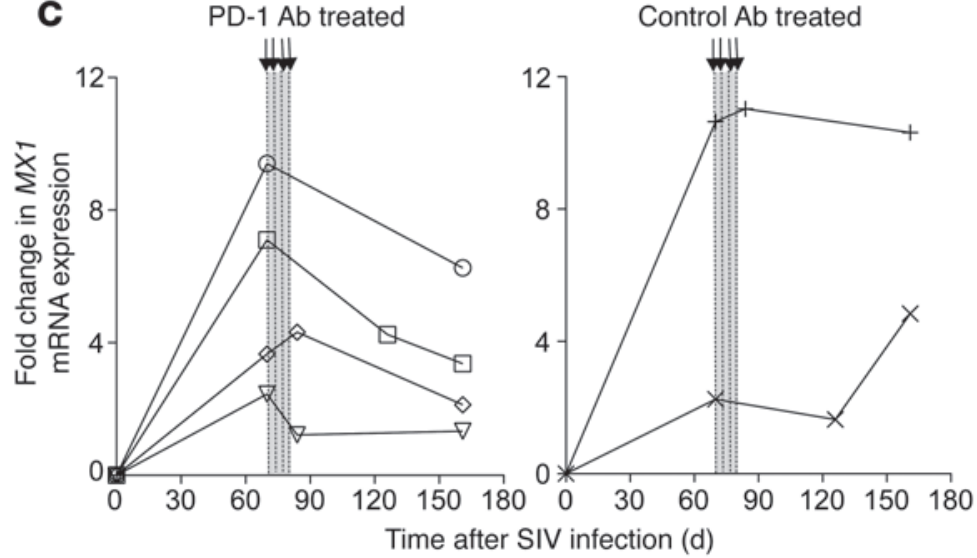

B Gut

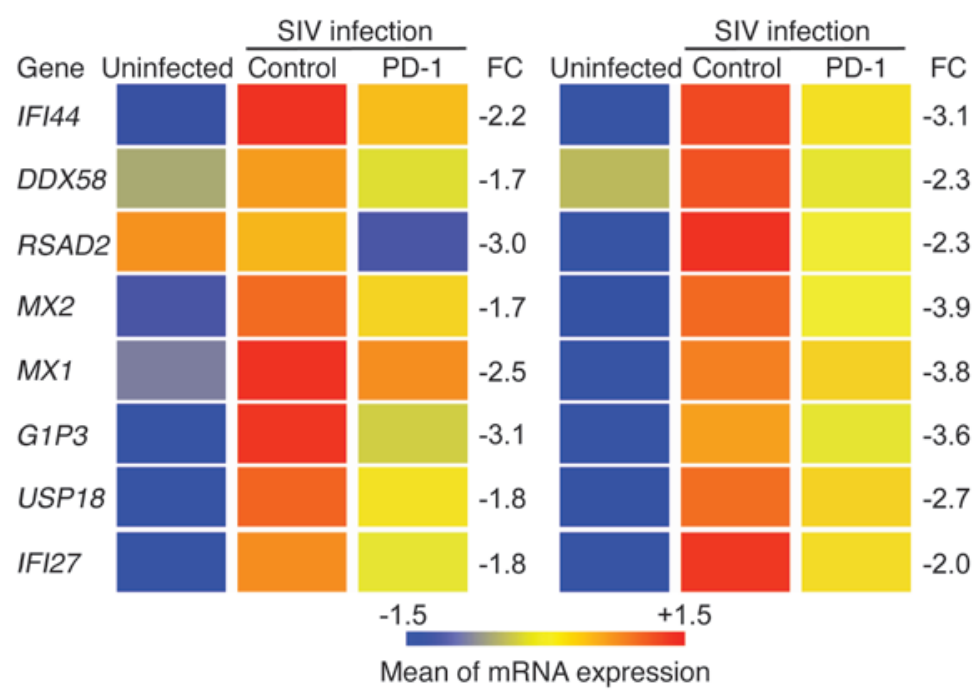

Mean of mRNA expression

Blood

D Day 56 after blockade

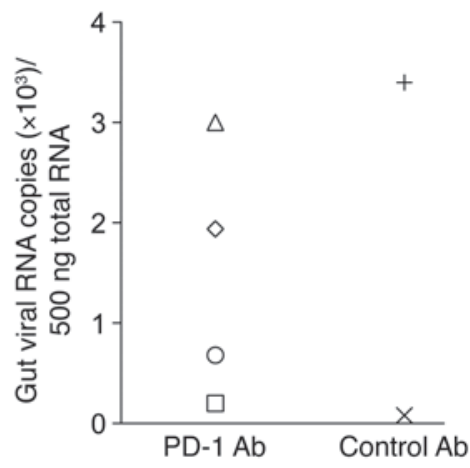

\section{Figure 1}

PD-1 blockade downregulates type I IFN responses in SIV-infected RMs. (A) IPA of differentially regulated genes $(P<0.05$ and $>1.5$-fold change) in blood and colorectum (Gut) of SIV-infected RMs at 14 days following PD-1 blockade. Percentage of genes that were up- or downregulated in a pathway in the PD-1 antibody-treated $(n=5)$ compared with control antibody-treated group $(n=3)$ are shown. (B) Heat maps of ISGs in SIVnegative (Uninfected; $n=4$ ), control antibody-treated (Control, $n=3$ ), and PD-1 antibody-treated (PD-1, $n=5)$ animals that were significantly downregulated at 14 days following PD-1 blockade. Mean mRNA expression for each group is shown. The fold changes (FC) in the PD-1 group compared with control group are shown. (C) qPCR analysis of MX1 mRNA in gut tissue of early chronic animals. Fold changes represent fold increase relative to preinfection value of the same RM. Arrows indicate the time of antibody treatments. (D) SIV RNA copies in the gut of PD-1and control antibody-treated early chronic RMs. ${ }^{*} P<0.05$.

We next performed quantitative real-time PCR (qPCR) analysis to study the mRNA expression kinetics of one of the significantly modulated ISGs, $M X 1$, in the gut using longitudinal samples from the same macaque before and after infection, and following PD-1 blockade (Figure 1C). These analyses revealed a strong increase in $M X 1$ transcripts following SIV infection and significant downregulation following PD-1 blockade that was evident even at 90 days (last point of analysis) following blockade. As expected, no significant reduction in $M X 1$ levels was observed in control antibodytreated animals. This sustained reduction in immune activation in PD-1 antibody-treated animals was not due to decreased SIV levels, as the viral RNA levels were comparable in the gut between antiPD-1 antibody-treated and control antibody-treated animals (Figure 1D) and remained relatively high in the plasma (Supplemental Figure 3). These results demonstrate that in vivo PD-1 blockade reduces expression of SIV-induced ISGs independent of virus levels, a condition that is normally seen in chronically SIV-infected sooty mangabeys (12) and African green monkeys (13).

Sustained proinflammatory responses could lead to changes in tight junction gene expression and alter the gut permeability barrier (15). So, we next investigated whether in vivo PD-1 blockade influenced tight junction-associated gene expression and gut permeability barrier. First, we analyzed the expression of genes associated with tight junctions in our microarray analyses. We found that transcripts encoded by genes associated with tight junctions, such as claudin 5 (CLDN5), junction adhesion molecule 2 (JAM2), connexin-45 (Cx45), and connexin-43 (Cx43) increased following PD-1 blockade (Figure 2A). We then confirmed the upregulation of transcripts specific for JAM2, CLDN5, and Cx45 using qPCR analysis, which showed enhanced expression of these genes fol- 
A

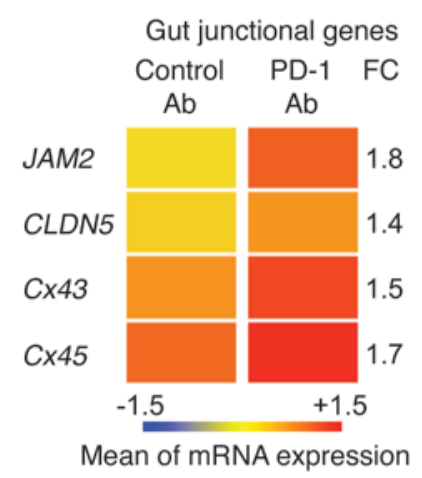

C

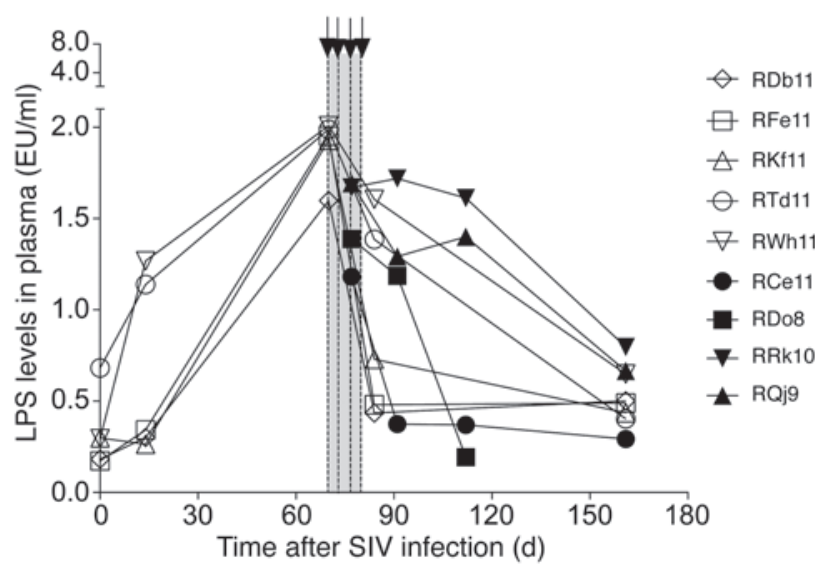

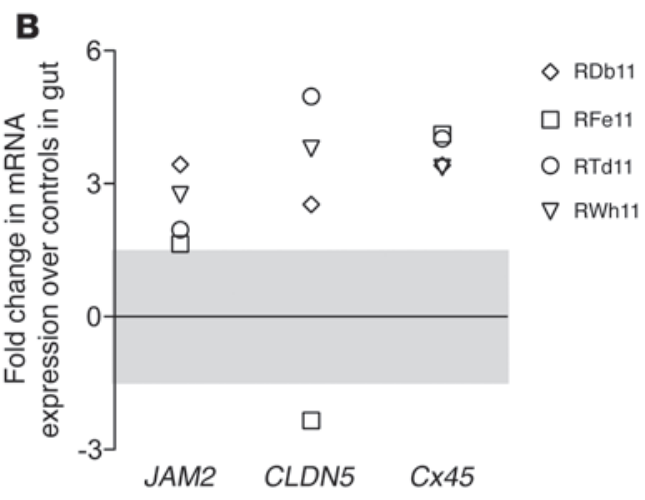

Control Ab treated

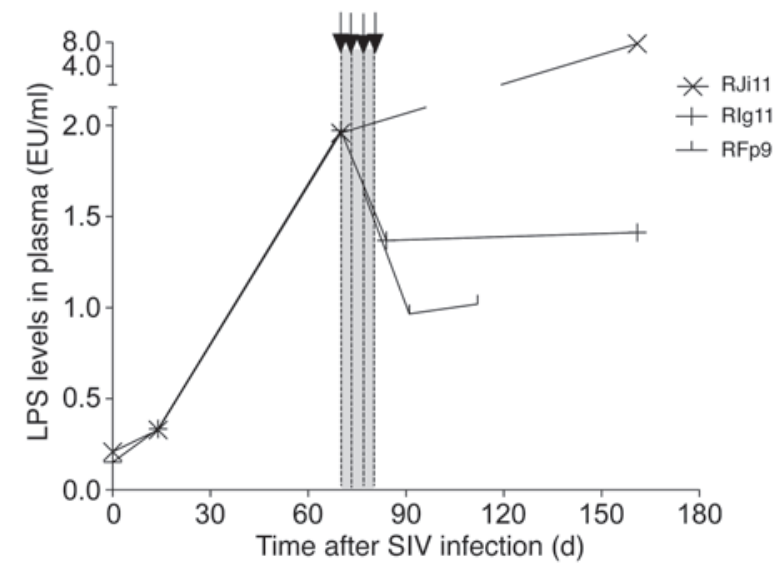

Figure 2

PD-1 blockade enhances gut junction-associated gene expression and reduces microbial translocation in SIV-infected RMs. (A) Heat map of junction-associated genes in the control antibody-treated $(n=3)$ and PD-1 antibody-treated $(n=5)$ animals that were significantly upregulated at 14 days following PD-1 blockade. Mean mRNA expression for each group is shown. The fold changes in the PD-1 group compared with the control antibody-treated group are shown. (B) qPCR analysis of CLDN5, JAM2, and Cx45 junctional genes in gut tissue. Fold changes represent fold increase relative to the average expression in 6 SIV-infected control RMs. (C) LPS levels in plasma in PD-1 antibody- and control antibody-treated SIV-infected RMs. Arrows indicate the time of antibody treatments. The LPS levels in the PD-1 group were significantly lower $(P=0.009$; Wilcoxon rank-sum test) than in the control group at 90 days following blockade.

lowing PD-1 blockade (Figure 2B). It is important to note that all of these genes have been shown to improve gut permeability barrier function (16-18). These results demonstrate that in vivo PD-1 blockade induces expression of some of the important tight junction genes during chronic SIV infection and suggest an increase in gut permeability barrier function. The mechanisms by which PD-1 blockade induces the expression of tight junction genes are not clear. We speculate that the reduced proinflammatory responses could have contributed to enhanced survival of gut epithelial cells. Alternately, PD-1 blockade could directly influence gut epithelial cells, as these cells express one of the PD-1 ligands, PD-L1 (1).

Previous studies demonstrated an association between decreased gut permeability barrier function and microbial translocation measured as increased LPS levels in plasma $(19,20)$; so, we measured LPS levels in plasma following SIV infection and PD-1 blockade. As expected, we observed a significant increase in plasma LPS levels following SIV infection, and we found a dramatic decrease in plasma LPS levels as early as 14 days following PD-1 blockade (Figure 2C). By 90 days following blockade, the plasma LPS in all animals reached preinfection levels. We could not follow LPS levels longterm in some of the control antibody-treated animals because they progressed to AIDS and were euthanized. However, in two of the control antibody-treated animals, we did not observe a significant decrease in LPS levels. These results suggest that in vivo PD-1 blockade during chronic SIV infection not only restores gut permeability barrier function but also reduces microbial translocation.

Reduced microbial translocation into the blood could also result from reduced microbial burden in the gut. Since PD-1 blockade can enhance the function of immunity against persistent antigens (1), we investigated the effect of in vivo PD-1 blockade on cellular and humoral immunity in blood against the gut-resident pathogens. Specifically, we studied the antibody and $\mathrm{CD}^{+} \mathrm{T}$ cell responses against Campylobacter, one of the common gut-resident bacteria in our macaque colony. The Campylobacter-specific antibody titers increased significantly (>2-fold) in sera of 5 of the 9 PD-1 antibody-treated animals at 90 days following blockade (Figure 3A). Similarly, Campylobacter-specific $\mathrm{CD}^{+} \mathrm{T}$ cell levels increased in 6 of the 9 PD- 1 antibody-treated animals by 90 days following blockade (Figure 3B). A similar increase was also observed for Salmonella-specific (another gut-resident bacterium) $\mathrm{CD}^{+} \mathrm{T}$ cells in the blood following PD-1 blockade (Figure 3B). Control RMs did not show any increase in $\mathrm{CD}^{+} \mathrm{T}$ cell responses against Campylobacter or Salmonella, but rather we observed a gradual decrease 
A

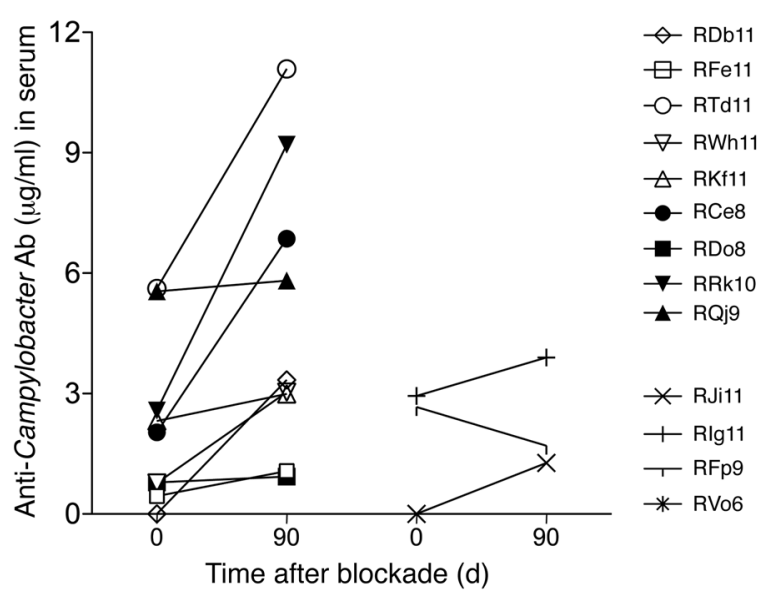

C

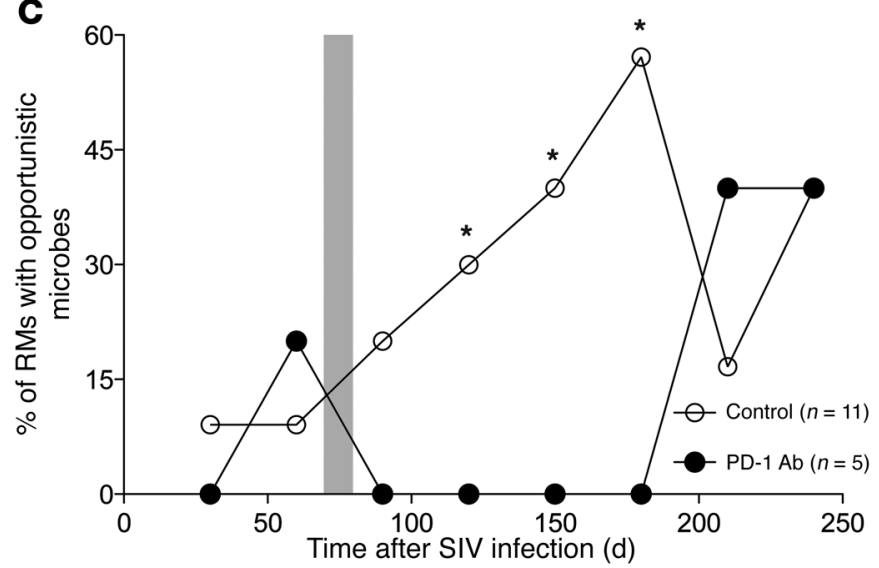

B

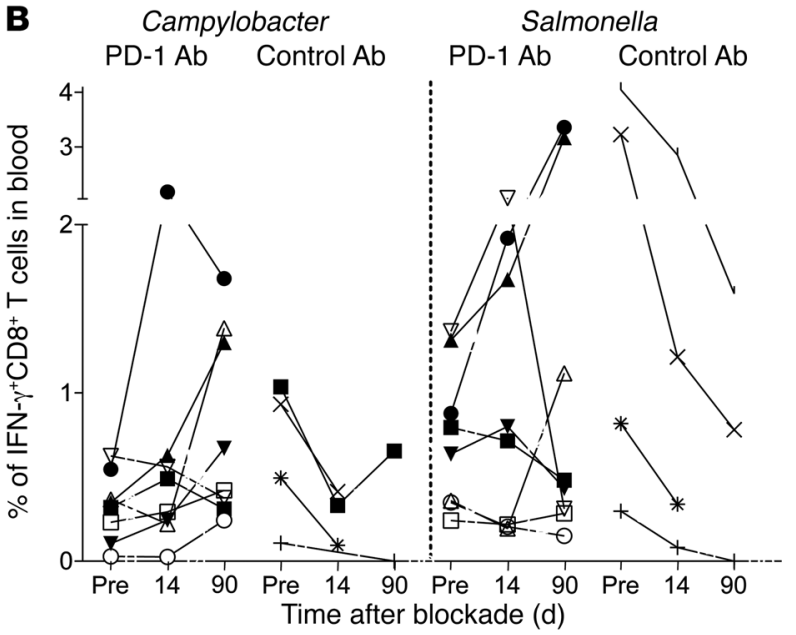

D

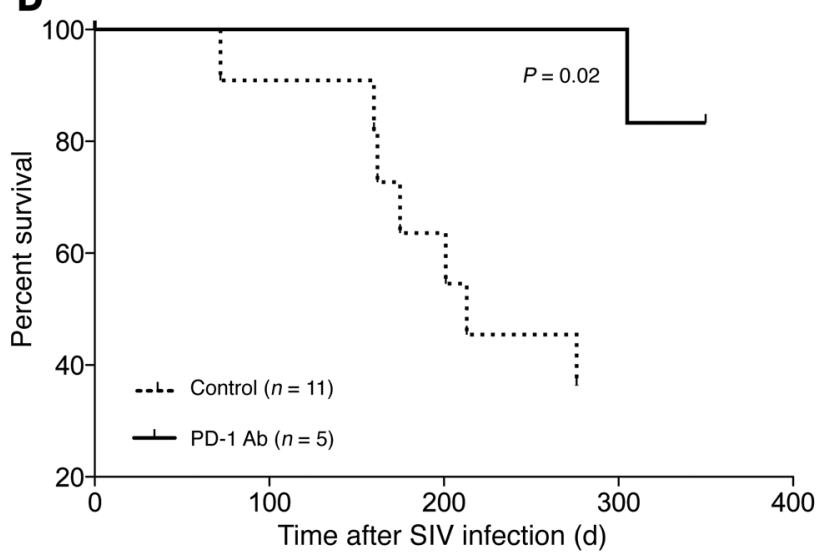

Figure 3

In vivo PD-1 blockade enhances immunity to pathogenic gut bacteria, decreases occurrence of opportunistic infections, and prolongs survival of SIV-infected RMs. (A) Anti-Campylobacter antibody titers in serum. (B) IFN- $\gamma^{+}$CD8+ T cell responses in blood. Pre, 0-25 days prior to initiation of antibody therapy. IFN- $\gamma^{+} \mathrm{CD} 8^{+} \mathrm{T}$ cell responses in the PD-1 group at 14 days and 90 days following blockade were significantly greater than in the control group $(P<0.01$; Wilcoxon rank-sum test). (C) Incidence of non-SIV-related opportunistic infections in controls (8 no antibody-treated and 3 control antibody-treated) or PD-1 antibody-treated (PD-1) SIV-infected early chronic RMs. See Supplemental Tables 1 and 2 for details about the nature of opportunistic infections. Shaded region indicates the period of antibody treatments. ${ }^{*} P=0.01, t$ test. (D) Kaplan-Meier survival plot of SIV-infected RMs shown in C. The 8 SIV-infected RMs in the "no antibody" control group were selected based on set point plasma viral load (between $10^{6}$ and $10^{7}$ ) to match with the set point viral load in the early chronic PD-1 group at the initiation of PD-1 blockade.

in $\mathrm{T}$ cell responses in these animals. These results showed that the enhanced immune responses against gut-resident pathogens could have contributed to the reduced immune activation and microbial translocation following PD-1 blockade.

To further confirm the reduced microbial burden in the gut, we followed the occurrence of opportunistic microbial infections such as Campylobacter, Cryptosporidium, Shigella, and Trichuris in stool samples of the 5 early chronic PD-1 antibody-treated RMs following SIV infection and PD-1 blockade, compared with the 3 early chronic control antibody-treated and 8 early chronic "no antibody"-treated SIV-infected RMs with comparable set point viral load (Figure 3C, Supplemental Figure 3, and Supplemental Tables 1 and 2). For these analyses, we excluded the late chronic animals, because members of this group naturally had a low incidence of opportunistic infections, with lower set point viremia and better preservation of immune function as evidenced by longer survival. About $10 \%-20 \%$ of the animals in the control group developed one or more opportunistic infections by 3 months after SIV infection, with about $40 \%$ developing infections by
5 months. Similarly, about $20 \%$ of the animals in the PD- 1 group were positive for opportunistic infection(s) at 2 months after SIV infection ( 2 weeks before blockade). However, following blockade, all animals in the PD-1 group remained negative for opportunistic infection(s) for about 5 months, demonstrating that in vivo PD-1 blockade enhanced the control of opportunistic infections in the gut.

Hyperimmune activation has been shown to be one of the strong predictors of disease progression, and the reduction of hyperimmune activation by the anti-PD- 1 antibody treatment could contribute to enhanced survival. Consistent with this hypothesis, animals in the PD-1 group survived significantly longer than the animals in the control group following SIV infection (Figure 3D). About 55\% (6 of 11) of the animals in the control group died by 270 days after SIV infection, whereas all 5 animals in the early chronic PD-1 group were alive at this time, demonstrating that in vivo PD-1 blockade enhanced the survival of SIV-infected RMs despite high viremia. This enhanced survival persisted even at 350 days after SIV infection (time of euthanasia to terminate the study), at 
which time 4 of 5 animals in the early chronic PD-1 group had survived. For the data presented in Figure 3D, we did not include data from the remaining 4 late chronic PD-1-treated animals for the reasons discussed above. However, none of these 4 late chronic PD-1-treated animals showed any evidence of opportunistic infections following blockade, and all survived for more than 200 days following PD-1 blockade (data not shown).

In conclusion, our results reveal an unanticipated but critical finding that a short treatment (10 days) with anti-PD-1 antibody during chronic SIV infection reduces hyperimmune activation and microbial translocation even under conditions of high viremia. More importantly, the reduced hyperimmune activation was associated with enhanced survival of SIV-infected RMs. These results are similar to what is seen in nonprogressive SIV infection in naturally infected hosts $(12,13)$ and HIV infection in viremic nonprogressors (21). The effects of PD-1 blockade on reducing hyperimmune activation could be a result of a combination of enhanced immunity against gut-resident pathogenic bacteria and repair of gut permeability barrier function. These results could aid in the development of novel therapeutic strategies to treat HIV/AIDS. For example, it may be that combination antiretroviral therapy in humans reduces hyperimmune activation to just a limited extent (22) because immunity against opportunistic infections is only partially restored. Our results suggest that combining PD-1 blockade with highly active antiretroviral therapy (HAART) may improve the benefits of HAART by enhancing immunity against opportunistic infections and reducing microbial translocation and hyperimmune activation.

\section{Methods}

Study group. SIV-infected RMs treated with either anti-PD-1 antibody or control antibody were described previously (8). Briefly, SIV-infected RMs were treated with therapeutic antibodies at either 10 or 90 weeks after SIV infection. Eight more SIV-infected macaques without any antibody treatment were used for some analyses. Macaques were infused with mouse anti-human PD-1 antibody or a control antibody at $3 \mathrm{mg} / \mathrm{kg}$ body weight on $0,3,7$, and 10 days. See Supplemental Methods for more details about animals, animal care, SIV infection, and anti-PD-1 antibody.

1. Sharpe AH, Wherry EJ, Ahmed R, Freeman GJ. The function of programmed cell death 1 and its ligands in regulating autoimmunity and infection. Nat Immunol. 2007;8(3):239-245.

2. Barber DL, et al. Restoring function in exhausted CD8 T cells during chronic viral infection. Nature. 2006;439(7077):682-687.

3. Day CL, et al. PD-1 expression on HIV-specific T cells is associated with T-cell exhaustion and disease progression. Nature. 2006;443(7109):350-354.

4. Trautmann L, et al. Upregulation of PD-1 expression on HIV-specific CD8+ T cells leads to reversible immune dysfunction. Nat Med. 2006; 12(10):1198-1202.

5. Petrovas C, et al. PD-1 is a regulator of virus-specific CD8+ T cell survival in HIV infection. J Exp Med. 2006;203(10):2281-2292.

6 . Velu V, et al. Elevated expression levels of inhibitory receptor programmed death 1 on simian immunodeficiency virus-specific CD8 T cells during chronic infection but not after vaccination. J Virol. 2007; 81(11):5819-5828

7. Petrovas C, et al. SIV-specific CD8+T-cells express high levels of PD1 and cytokines but have impaired proliferative capacity in acute and chronic SIVmac251 infection. Blood. 2007;110(3):928-936.

8. Velu V, et al. Enhancing SIV-specific immunity in vivo by PD-1 blockade. Nature. 2009;458(7235):206-210.
LPS levels. Plasma samples were quantified using a limulus amebocyte assay (Lonza) according to the manufacturer's protocol. See Supplemental Methods for details.

qPCR analysis. $M X 1$ gene expression and tight junction gene expression were measured using SYBR green assays as described previously (12) with primer sequences listed in Supplemental Table 3. See Supplemental Methods for details.

Cellular and humoral responses. The titer of anti-Campylobacter IgG was detected as described previously for SIV-specific IgG (9), except that Campylobacter protein $(2 \mu \mathrm{g} /$ well $)$ was used as coating antigen. T cell cytokine profiles were determined following stimulation with Campylobacter $(5 \mu \mathrm{g})$ or Salmonella (0.5 $\mu \mathrm{g})$ lysates as described previously (8). See Supplemental Methods for details.

Microarray data accession number. Microarray results have been deposited in the Gene Expression Omnibus database (GEO GSE33933).

Statistics. Significance of gene regulation according to microarray analysis was determined by 1-way ANOVA test. Significance of changes in LPS levels and cellular immune responses were determined by Wilcoxon rank-sum test; opportunistic infections by a 2-tailed $t$ test; and survival by MantelCox log rank test. For all tests, a $P$ value less than 0.05 was considered significant. See Supplemental Methods for more details.

Study approval. Macaques were housed at the Yerkes National Primate Research Center and were cared for under the guidelines established by the Animal Welfare Act and the NIH Guide for the Care and Use of Laboratory Animals (publication no. 85-23. Revised 1985) and studied under protocols approved by the Emory University IACUC.

\section{Acknowledgments}

We thank R. Ahmed for critical input throughout the study and the veterinary staff at the Yerkes National Primate Research Center for animal care. See Supplemental Acknowledgments for more details.

Received for publication August 22, 2011, and accepted in revised form February 8, 2012.

Address correspondence to: Rama Amara, The Yerkes National Primate Research Center, Emory University, 954 Gatewood Road, Atlanta, Georgia 30329, USA. Phone: 404.727.8765; Fax: 404.727.7768; E-mail: ramara@emory.edu.
9. Titanji K, et al. Acute depletion of activated memory $\mathrm{B}$ cells involves the PD-1 pathway in rapidly progressing SIV-infected macaques. J Clin Invest. 2010;120(11):3878-3890.

10. Finnefrock AC, et al. PD-1 blockade in rhesus macaques: impact on chronic infection and prophylactic vaccination. J Immunol. 2009;182(2):980-987.

11. Abel K, Alegria-Hartman MJ, Rothaeusler K, Marthas M, Miller CJ. The relationship between simian immunodeficiency virus RNA levels and the mRNA levels of alpha/beta interferons (IFN-alpha/beta) and IFN-alpha/beta-inducible Mx in lymphoid tissues of rhesus macaques during acute and chronic infection. JVirol. 2002;76(16):8433-8445.

12. Bosinger SE, et al. Global genomic analysis reveals rapid control of a robust innate response in SIVinfected sooty mangabeys. J Clin Invest. 2009; 119(12):3556-3572.

13. Jacquelin B, et al. Nonpathogenic SIV infection of African green monkeys induces a strong but rapidly controlled type I IFN response.J Clin Invest. 2009; 119(12):3544-3555.

14. Quigley M, et al. Transcriptional analysis of HIVspecific CD8+ T cells shows that PD-1 inhibits T cell function by upregulating BATF. Nat Med. 2010; 16(10):1147-1151.

15. Zeissig $\mathrm{S}$, et al. Changes in expression and distribution of claudin 2,5 and 8 lead to discontinuous tight junctions and barrier dysfunction in active Crohn's disease. Gut. 2007;56(1):61-72.

16. Ey B, Eyking A, Gerken G, Podolsky DK, Cario E. TLR2 mediates gap junctional intercellular communication through connexin-43 in intestinal epithelial barrier injury. J Biol Chem. 2009;284(33):22332-22343.

17. Amasheh $\mathrm{S}$, et al. Contribution of claudin-5 to barrier properties in tight junctions of epithelial cells. Cell Tissue Res. 2005;321(1):89-96.

18. Ueki T, Iwasawa K, Ishikawa H, Sawa Y. Expression of junctional adhesion molecules on the human lymphatic endothelium. Microvasc Res. 2008; 75(2):269-278.

19. Brenchley JM, et al. Microbial translocation is a cause of systemic immune activation in chronic HIV infection. Nat Med. 2006;12(12):1365-1371.

20. Estes JD, et al. Damaged intestinal epithelial integrity linked to microbial translocation in pathogenic simian immunodeficiency virus infections. PLoS Pathog. 2010;6(8):e1001052.

21. Rotger $\mathrm{M}$, et al. Comparative transcriptomics of extreme phenotypes of human HIV-1 infection and SIV infection in sooty mangabey and rhesus macaque. J Clin Invest. 2011;121(6):2391-2400.

22. Cassol E, et al. Persistent microbial translocation and immune activation in HIV-1-infected South Africans receiving combination antiretroviral therapy. J Infect Dis. 2010;202(5):723-733. 